RU Современные LMS платформы дистанционного обучения: анализ и сравнение

Исаева Е. С.

\begin{abstract}
Аннотация. Целью данного исследования является определение основных параметров и инструментов систем управления обучением (англ. learning management system, LMS), выявление сильных и слабых сторон на примере нескольких платформ дистанционного образования (Moodle, Google Classroom, Microsoft Teams, Open edX и ILIAS), широко применяемых не только в России, но и во всем мире, с целью обнаружения педагогического потенциала для онлайн-обучения в высших учебных заведениях. В данной статье перечислены основные элементы информационно-технологической поддержки обучения, сделаны выводы о наиболее эффективных LMS платформах. Научная новизна заключается в выявлении основных характеристик платформ, необходимых для успешной реализации онлайн-образования в современных условиях. В результате исследования выделены основные параметры и инструменты LMS систем.
\end{abstract}

\title{
Modern LMS Platforms for Distance Education: Analysis and Comparison
}

\author{
Isaeva E. S.
}

Abstract. The study aims to determine the main parameters and tools of learning management systems (LMS), identify their strengths and weaknesses using the example of several distance education platforms (Moodle, Google Classroom, Microsoft Teams, Open edX and ILIAS), which are widely used not only in Russia but also around the world, in order to bring out the pedagogical potential for online learning at higher education institutions. The paper lists the main elements of information technology support for learning, draws conclusions about the most effective LMS platforms. Scientific novelty lies in identifying the main characteristics of the platforms that are necessary for the successful implementation of online education in current conditions. As a result of the study, the researcher has determined the main parameters and tools of LMS.

\section{Введение}

Актуальность. С развитием новых информационных технологий в последние годы демонстрируется технологическое совершенствование виртуальных образовательных сред. Этот факт подталкивает на использование новых инструментов, предложение новых педагогических подходов, которые способствовали бы самостоятельной работе обучающихся.

В последние десятилетия классическая модель обучения претерпела многочисленные изменения. Появление Интернета ускорило эти изменения благодаря своей способности предлагать различные возможности подхода к информации, основанного на динамичных технологиях. Можно с уверенностью сказать, что Интернет - это посредник в доступе к образованию и культуре, в то время как онлайн-обучение - это новая форма образования, которая предлагает себя в качестве альтернативы в непрерывном обучении и приобретении знаний. Большим преимуществом электронного обучения является отмена формальных барьеров путем исключения расстояний и введения временной гибкости (Тихомиров, 2011).

Электронное обучение представляет собой связь между средой информационно-коммуникационных технологий и средой образования. Понимание этого лежит в основе хорошо спланированной и организованной учебной среды, но ни в коем случае такое обучение не может заменить существующие педагогические модели и стандарты. Электронное обучение - это инструмент, предоставляемый информационными технологиями для поддержки обучения. Другими словами, оно расширяет возможности с целью приобретения знаний.

Электронное обучение играет немаловажную роль в современной жизни, выполняя ряд задач, начиная от предоставления конкретных знаний, заканчивая профессиональной подготовкой, не только для студентов в привычном формате обучения, но и для всех категорий участников обучения (обучение во время работы,

Научная статья (original research article) | https://doi.org/10.30853/ped20210127

(๔) 2021 Авторы. 000 Издательство «Грамота» (๔ 2021 The Authors. GRAMOTA Publishers). Открытый доступ предоставляется на условиях лицензии СС ВY 4.0 (open access article under the CС BY 4.0 license): https://creativecommons.org/licenses/by/4.0/ 
получение квалификации для новых профессий). Таким образом, применение дистанционного обучения вносит свой вклад в развитие образования в стране и в первую очередь для людей с ограниченными возможностями. Несмотря на явные плюсы дистанционного обучения, существуют и явные проблемы, связанные с тем, что большинство участников не имеют практического опыта работы с данной формой обучения: неумение студентов планировать время; ограниченный личный контакт с преподавателем; требовательная подготовка учебных материалов (Кузнецов, 2019).

Принимая во внимание проблемы дистанционного обучения, мы считаем, что крайне важно, чтобы образовательные учреждения работали над развитием данного направления, благодаря чему изменится современная парадигма образования и отношение к этой методике обучения. В этом контексте разработка новых и эффективных методов обучения становится актуальной для формирования общества, нацеленного на знания, для чего требуется нечто большее, чем просто обучение, изучение учебников и сдача экзаменов (Батаев, 2017).

Для проведения обучения через Интернет используют технологии Web 2.0, содержащие в себе различные инструменты и сервисы, такие как вики, блоги, обмен мгновенных сообщений, социальные сети, вся необходимая для обучения справочная информация, которые не только дают новые возможности преподавателю создавать и распространять обучающий контент, но и способствуют появлению новых способов взаимодействия между студентом и преподавателем.

Немаловажную роль в электронном обучении играет дизайн. Успешное электронное обучение основывается на балансе повышения качества образования и его построения. Невозможно развивать одно и пренебрегать другим.

Система управления обучением должна быть открытой, хорошо спроектированной с точки зрения дизайна, адаптивной, с меньшим количеством барьеров и большей инклюзивностью. Таким образом, платформы LMS приобретают очевидное значение, поскольку успех электронного обучения напрямую зависит от его построения. В учебном процессе важно, чтобы учащийся достиг максимального понимания передаваемой информации, которую он мог бы применять на практике (Петрова, 2019). Платформы способны сделать процесс обучения проще и интереснее. Для этого необходимо определить, какая платформа будет отвечать всем этим требованиям, предлагает ли она инструменты для самостоятельного развития, чтобы учащийся смог осознать, что он сам развивает свои знания, тем самым формируя больше ответственности.

Задачи исследования:

- изучить основные характеристики и инструменты современных LMS систем;

- выявить и сравнить основные инструменты платформ, отвечающие принципам обучения.

В основу исследования легли научные теории и методы анализа Г. Файрбоу (Firebaugh, 2008), А. Суфиан (Sufian, 2015); теоретические и практические аспекты онлайн-обучения, рассмотренные А. В. Батаевым (2017), М. Ю. Глотовой и Е. А. Самохваловой (2015), Н. В. Кузнецовым (2019), Ю. Н. Мироновой (2020), В. И. Петровой (2019), П. Д. Рабиновичем, К. Е. Заведенским, М. Э. Кушниром и др. (2020), В. П. Тихомировым (2011), Т. Д. Аллен, Т. Д. Голденом, К. М. Шокли (Allen, Golden, Shockley, 2015), Хувен К., Зооненом У. (Hоеvеn, Zoonen, 2015), Сэлимом X. M. (Selim, 2007); теоретические подходы к построению учебного процесса, представленные В. А. Сластениным, И. Ф. Исаевым, Е. Н. Шияновой (2002).

Метод исследования: теоретический (анализ и изучение соответствующей научной литературы, анализ ресурсов систем дистанционного обучения).

Практическая значимость исследования состоит в возможности дальнейшего применения полученных результатов в реализации как смешанного, так и дистанционного обучения в образовательных учреждениях.

Основываясь на методах тематического исследования, предложенных в литературе (Firebaugh, 2008; Sufian, 2015), постараемся ответить на вышеобозначенные вопросы, проводя сравнительное исследование наиболее популярных платформ дистанционного обучения.

\section{Основные характеристики и инструменты современных LMS систем}

Для облегчения создания учебной среды доступны различные платформы. В них заложены технологические и педагогические инструменты для разработки методик обучения с использованием каналов вебвзаимодействия, способных оказывать поддержку образовательной деятельности виртуальным образом.

LMS представляет собой программное приложение, которое обеспечивает контроль над процессом обучения и преподавания. Такая система дает возможность регистрировать студентов, упорядочивать курсы в каталоге, отслеживать успехи студентов и сообщать обо всем, что было сделано преподавателю или куратору, а также позволяет не только создавать и хранить материалы, но и использовать их повторно в разных предметных областях знаний и разными учащимися. К таким многоразовым объектам можно отнести текст, презентации, анимации, картинки (Исаева, 2017; Selim, 2007).

Нами были выбраны платформы, наиболее цитируемые в исследовательских источниках и используемые вузами страны: Moodle, Google Classroom, Microsoft Teams, а также менее известные в России - Open edX и ILIAS.

С целью выявления их различий были установлены критерии анализа: распространение, совместная работа, интерактивность, мультимедиа, удобство использования и доступность.

Поиск на веб-сайтах крупнейших вузов страны показал, что реальное использование технологий дистанционного образования изменилось с 2020 года. Практически все вузы на данный момент задействуют 
в образовательной деятельности современные площадки, единственное, сами учреждения не предоставляют информацию о типе платформы.

На данный момент наиболее популярной платформой во всем мире для электронного образования является LMS Moodle, ведущие вузы страны активно используют данную платформу в качестве информационно-образовательной среды. По официальным данным, на сегодняшний день зарегистрировано 5680 сайтов на платформе Moodle. Далее приведен анализ выбранных платформ:

- Moodle (https://moodle.org)

Бесплатное программное обеспечения для поддержки обучения, разработанное на языке программирования php и с системой управления базами данных MySQL, PostgreSQR, Oracle, Assess, Interbase. Предоставляется как ПО с открытым исходным кодом под стандартной общественной лицензией GNU General Public License, пользователи которого имеют право на неограниченную установку, использование, распространение и изменение Moodle без каких-либо лицензионных сборов. Имеет множество инструментов как для самостоятельной, так и для совместной работы студентов, что позволяет создавать свою собственную образовательную платформу. Совместная работа организуется с помощью таких инструментов, как вики (позволяет учащимся создавать совместный документ подобно Википедии), форумы (позволяет студентам и преподавателям обмениваться идеями, файлами), семинары (инструмент, позволяющий учащимся оценивать работы друг друга), имеется возможность проводить видеоконференции (Глотова, Самохвалова, 2015), а поддержка SCORM позволяет создавать интерактивные занятия. Moodle включает в себя множество способов измерения (Задания, Тест) и отслеживания успеваемости учащихся (Журнал). Позволяет награждать и поощрять учащихся (Значки, Сертификаты). Moodle LMS соответствует стандарту WCAG 2.1 AA (рекомендации по доступности вебконтента), представляет собой набор рекомендаций по повышению доступности веб-контента, в первую очередь для людей с ограниченными возможностями (https://www.iso.org/standard/58625.html).

- Google Classroom (https://edu.google.com)

Разработчиком данного веб-сервиса является американская транснациональная корпорация Google, которая стремится упростить процесс обмена файлами между учителями и учениками. Как сообщается на вебсайте, Classroom - это бесплатная система, которая объединяет такие инструменты, как Документы Google, Диск и почтовый сервис Gmail. Для полноценной работы преподавателя и учащихся учебному заведению нужно запросить лицензию Google Workspace for Education. Данная платформа предоставляет возможности для создания заданий, оставления отзывов и общения со студентами в режиме реального времени. По сравнению c Moodle данная платформа имеет меньшее количество инструментов, что компенсируется простотой использования, расширение функционала возможно с помощью дополнительных инструментов Google. Совместная работа осуществляется посредством инструмента Задания, который размещается в виде прикрепленного файла, ссылки или в форме опроса. Существенным минусов данной системы является отсутствие вебинарной комнаты, видеоконференции можно проводить только с помощью сервиса Google Meet. Также отсутствуют какие-либо интерактивные инструменты. Для оценивания и отслеживания прогресса предусмотрены инструменты Задания, Журнал. Расширенная версия Google Workspace имеет необходимые инструменты, помогающие адаптировать систему под нужды всех учащихся, в том числе с нарушением зрения или слуха, с ограниченными физическими возможностями.

- Microsoft Teams (https://www.microsoft.com)

Microsoft Teams является частью пакета Office 365 и распространяется по корпоративной подписке, предназначена для конференц-связей, командной работы, дистанционного обучения. Данная платформа очень похожа на Google Classroom, содержит такие же инструменты для совместной работы, Задания, Тесты. Отличительной особенностью данной системы от предыдущей являются встроенный инструмент для видеовстреч, возможность осуществлять как групповые видео- и аудиозвонки, так и личные. То же самое касается и инструмента Чат, который делится на групповой и личный. В сервис встроены все офисные приложения, которыми можно пользоваться, не загружая себе на компьютер. B Microsoft Tеams имеется общее рабочее пространство, в котором одновременно могут работать все студенты и преподаватели (Миронова, 2020). На официальном сайте Microsoft указано, что Майкрософт учитывает стандарт WCAG при разработке продуктов и служб, стремясь обеспечить доступность технологий и данных для всех клиентов. Какие конкретно используются инструменты для обеспечения доступности среды, в Microsoft Teams не указано. В самой же системе были обнаружены такие функции, как субтитры, иммерсивное средство чтения.

- Open edX (https://open.edx.org)

Это бесплатная образовательная платформа с открытым кодом от компании еdX, которая не уступает по своему функционалу LMS Moodle. Содержит ряд образовательных инструментов: видеоконференции, чат, обмен файлами, поддержка совместных проектов, оценивание, глоссарий, опросы, контроль доступа и т.д. Поддерживает SCORM - пакет разработки для учебных модулей, что позволяет создавать различные мультимедийные файлы, например презентации, онлайн-тренажеры. Интерфейс разработки курса содержит параметры для создания страниц для встраивания различных инструментов в эти страницы. Слушатели курса имеют возможность участвовать в активных обсуждениях, которые могут быть организованы как в рамках модуля курса, так и в виде обсуждений в рамках всего курса. Существует множество вариантов настройки, управления и модерации для обсуждений. Учащиеся могут легко получить доступ к платформе через браузер без необходимости установки какого-либо дополнительного программного обеспечения. Open edX имеет 
мобильное приложение, доступно для iOS и Android. В дополнение к образовательным инструментам, которые поддерживают обучение, общение, Open edX также предоставляет набор административных инструментов, помогающих автору в управлении и улучшении курса. Оценки учащихся и выполненные задания курса отслеживаются и отображаются для просмотра учащимися и преподавателями. По завершению курса имеется возможность предоставления сертификатов, шаблоны которых легко настраиваются. Все функции соответствуют стандарту доступности WCAG, для видео поддерживаются многоязычные субтитры.

- ILIAS (https://www.ilias.de)

Это система дистанционного обучения, разработанная в рамках проекта VIRTUS в Кёльнском университете в 1998 году и предоставляемая по лицензии GNU. Интерфейс, подобный социальной сети, позволяет обучающимся быстро разобраться и адаптироваться. Данная система позволяет создавать, управлять курсами, учебными модулями, проводить тесты и экзамены. Активно используется как платформа для смешанного обучения, в качестве дополнения к офлайн-занятиям, где студенты помимо теоретических материалов используют дискуссионные формы, тесты и различные совместные проекты. Позволяет контролировать доступ как к отдельным инструментам, так и к курсам в целом. По завершению курса обучающиеся имеют возможность получить сертификаты. Так же, как Moodle и Open edX, поддерживает SCORM-пакет. Недостатком данной платформы является отсутствие вебинарной комнаты, невозможность проведения онлайн-встреч. Не существует каких-либо справочников на русском языке, имеется техническая поддержка, но только на английском и немецком языках. Также нет информации о доступности платформы для лиц с ОВ3.

B Таблице 1 представлено сравнение основных характеристик LMS систем для реализации дистанционного обучения.

Таблица 1. Основные характеристики LMS систем, играющие роль в выборе платформы для реализации дистанционного обучения

\begin{tabular}{|c|c|c|c|c|c|}
\hline Характеристики & Moodle & $\begin{array}{l}\text { Microsoft } \\
\text { Teams }\end{array}$ & $\begin{array}{c}\text { Google } \\
\text { Classroom }\end{array}$ & Open edX & ILIAS \\
\hline Необходимость установки на сервер & + & - & - & + & + \\
\hline Возможность интеграции & + & + & - & + & + \\
\hline Удобство интерфейса & + & + & + & + & + \\
\hline Администрирование & + & + & + & + & + \\
\hline Адаптивность системы на любом устройстве & + & + & + & + & + \\
\hline Собственное приложение & + & + & + & + & - \\
\hline Техподдержка на русском языке & + & + & + & - & - \\
\hline Группировка обучающихся & + & + & + & + & + \\
\hline Уведомления пользователей & + & + & + & + & + \\
\hline Планирование & + & + & - & + & + \\
\hline Поддержка SCORM & + & - & - & + & + \\
\hline $\begin{array}{l}\text { Возможность разработки учебных материалов } \\
\text { внутри платформы }\end{array}$ & + & + & - & + & + \\
\hline Формирование отчетов & + & + & + & + & + \\
\hline Автоматическое оценивание & + & + & - & + & + \\
\hline Установка ограничений по времени & + & - & - & + & + \\
\hline Доступность для лиц с ОВЗ & + & + & + & + & - \\
\hline
\end{tabular}

На основании вышеизложенного, можно сделать вывод, что использование некоторых рассматриваемых платформ, таких как Microsoft Teams и Google Classroom, не всегда возможно для практических занятий, но эти инструменты могут служить хорошим дополнением при реализации смешанного обучение.

Приложения Moodle, Open edX, ILIAS имеют более широкие возможности, что позволяет использовать данные платформы не только для проведения занятий в онлайн-режиме, но и полностью обеспечить дистанционное обучение.

\section{Педагогические принципы в организации учебного процесса с помощью LMS платформы}

Важно помнить, что использование современных образовательных технологий не отменяет проблем, присущих образовательной системе. К таким проблемам можно отнести низкую практическую направленность сферы образования, что приводит к неумению учащихся пользоваться полученной информацией, применять ее на практике. Создание эффективной онлайн-среды требует от преподавателя хорошей педагогической подготовки. Рассмотрим основные педагогические принципы (Сластенин, Исаев, Шиянова, 2002), применяемые для реализации дистанционного обучения.

Принцип научности обучения ориентирован на применение современных технологических инструментов, которые помогают активизировать познавательную активность обучающихся. С развитием новых информационных технологий в последние годы демонстрируется технологическое совершенствование виртуальных образовательных сред. Этот факт подталкивает к использованию новых инструментов, предложению новых педагогических подходов, которые способствовали бы самостоятельной работе обучающихся. 
Процесс обучения в виртуальной среде не должен ограничиваться передачей знаний, а должен быть расширен, что приведет к формированию навыков и умений, которые позволят решать интеллектуальные задачи, необходимые для профессионального будущего. В таком контексте нелегко определить необходимую платформу, содержащую в себе множество инструментов. Образовательная платформа не должна быть сложной в понимании, чтобы не стать препятствием для обучения, что соответствует принципу доступности. Участники должны иметь возможность пользоваться всеми инструментами, которые помогают в освоении материала, при этом предоставляют своей аудитории некоторые преимущества: обучение в профессиональной или домашней среде, самостоятельное планирование времени, доступ к платформе даже через мобильные устройства. Студенты, впервые посещающие онлайн-курсы, часто не знают, что эти курсы из себя представляют. Поэтому очень важно на начальном этапе дать разъяснения по использованию доступных инструментов, рекомендации о том, сколько времени они должны уделять онлайн-занятиям в течение недели.

Реализация принципа последовательности обучения достигается путем планирования тематических модулей согласно учебному плану с помощью таких инструментов, как Календарь, Планировщик. Геймификация, обратная связь, ответы на вопросы обучающихся - все это можно отнести к реализации принципа активности и мотивированности в обучении. Практические онлайн-тесты, интерактивные задания дают обратную связь автоматически, тем самым мотивируя обучающихся и закрепляя полученные знания. Еще один важный принцип - это возможность непрерывной взаимосвязи как между студентом и преподавателем, так и между студентами. Реализация принцила группового и индивидуального обучения достигается посредством взаимодействия и сотрудничества. В онлайн-обучении существует множество инструментов для реализации данного принципа. К ним относятся многочисленные дискуссионные форумы, чаты, использование форумов социальных сетей, а также инструменты для совместной работы, такие как вики, совместная работа с экраном, семинары (Hoeven, Zoonen, 2015). В Таблице 2 представлено сравнение систем LMS по основным инструментам, необходимым для успешной реализации обучения.

Таблица 2. Основные инструменты LMS платформ

\begin{tabular}{|c|c|c|c|c|c|}
\hline Инструменты & Moodle & $\begin{array}{c}\text { Microsoft } \\
\text { Teams }\end{array}$ & $\begin{array}{c}\text { Google } \\
\text { Classroom }\end{array}$ & Open edX & ILIAS \\
\hline Видеоконференция & + & + & + & + & - \\
\hline Глоссарий & + & - & - & + & + \\
\hline Вики & + & - & - & + & + \\
\hline Возможность создания интерактивных заданий & + & - & - & + & + \\
\hline Задания & + & + & + & + & + \\
\hline Тесты & + & + & - & + & + \\
\hline Форум & + & - & - & + & + \\
\hline Чат & + & + & + & + & + \\
\hline Журнал успеваемости & + & + & + & + & + \\
\hline Презентации & + & + & + & + & + \\
\hline Совместная работа с экраном & + & + & - & + & - \\
\hline Сертификаты & + & - & - & + & + \\
\hline
\end{tabular}

Совокупное действие рассмотренных принципов обучения обеспечивает правильное определение его задач, содержания, выбор форм и методов. В таблице были выделены основные инструменты, отвечающие принципам научности, доступности, последовательности, мотивированности, группового и индивидуального обучения. В результате чего можно сделать вывод, что все выбранные платформы отвечают данным принципам. Несмотря на это, видна разница в предложенных инструментах для совместного обучения, а также в плане интерактивности. Некоторые платформы имеют больше возможностей. Наиболее широкий выбор инструментов и настроек, как видно из сравнения, представляет нам платформы Moodle и Open edX. Основные инструменты Microsoft Teams и Google Classroom также отвечают принципам.

\section{Заключение}

В ходе исследования были изучены основные характеристики и инструменты LMS систем, в результате можно сделать вывод, что данные системы могут быть использованы для чтения лекций как гуманитарных, так и технических дисциплин (за счет возможности работы с текстом, звуком, мультимедиа). Главным критерием выбора платформы для технических дисциплин может стать, на наш взгляд, наличие SCORM-пакета, позволяющего создавать тренажеры, симмуляционные упражнения. В связи с этим использование некоторых систем не всегда возможно для проведения практических или лабораторных занятий. Были выделены основные параметры для выбора платформы как с технической точки зрения, так и с педагогической. Рассмотрены основные инструменты, отвечающие принципам обучения, на которые также необходимо обратить внимание для планирования и реализации процесса обучения с помощью LMS. Критериями выбора должна служить система показателей, включающая в себя основные характеристики и возможности платформы.

Анализ 5 платформ дистанционного обучения по этим критериям позволил сделать вывод, что LMS Moоdle на сегодняшний день является не только самой востребованной системой в России, но и технологически 
развитой, что обусловлено не только открытым кодом, но и большим функционалом, технической поддержкой, предлагаемой командой разработчиков. Полным аналогом системы Moodle является Open edX, использование которой только набирает обороты в учебных заведениях страны и требует дальнейшего изучения.

В результате проведенного исследования можно рекомендовать рассмотренные LMS системы как вспомогательный инструмент к традиционным методам преподавания, который в каких-то моментах даже может их заменить, тем самым повысив шансы на доступность образования.

Перспективы дальнейшего исследования заключаются в более детальном анализе малоизученных платформ, а также в разработке методических рекомендаций по применению дистанционных технологий в изучении конкретных дисциплин.

\section{Источники | References}

1. Батаев А. В. Электронное обучение как условие повышения качества подготовки инженерно-экономических кадров // Планирование и обеспечение подготовки кадров для промышленно-экономического комплекса региона. 2017. Т. 1.

2. Глотова М. Ю., Самохвалова Е. А. Индивидуальные образовательные траектории на базе систем дистанционной поддержки образовательного процесса на примере СДО Moodle // Наука и школа. 2015. № 5.

3. Исаева Е. С. Электронное обучение как способ организации самостоятельной работы студентов // Филологические науки. Вопросы теории и практики. 2017. № 1-2.

4. Кузнецов Н. В. Онлайн-образование: ключевые тренды и препятствия // E-Management. 2019. № 1.

5. Миронова Ю. Н. Использование дистанционных технологий при проведении занятий: LMS MOODLE, GOOGLE KЛACC, MICROSOFT TEAMS // Азимут научных исследований: педагогика и психология. 2020. T. 9. № 4 (33).

6. Петрова В. И. Организация и планирование самостоятельной работы студентов педагогического образования в контексте смешанного обучения // Проблемы современного педагогического образования. 2019. № 65-3.

7. Рабинович П. Д., Заведенский К. Е., Кушнир М. Э. и др. Цифровая трансформация образования: от изменения средств к развитию деятельности // Информатика и образование. 2020. № 5 (314).

8. Сластенин В. А., Исаев И. Ф., Шиянова Е. Н. Педагогика: учеб. пособие для студ. высш. пед. учеб. заведений / под ред. В. А. Сластенина. М.: Академия, 2002.

9. Тихомиров В. П. Мир на пути Smart education. Новые возможности для развития // Открытое образование. 2011. № 3.

10. Allen T. D., Golden T. D., Shockley K. M. How Effective Is Telecommuting? Assessing the Status of Our Scientific Findings // Psychological Science in the Public Interest. 2015. Vol. 16. Issue 2.

11. Firebaugh G. Seven rules for social research. Princeton: Princeton University Press, 2008

12. Hoeven C., Zoonen W. Flexible Work Designs and Employee Well-being: Examining the Effects of Resources and Demands // New Technology, Work and Employment. 2015. Vol. 30. Issue 3.

13. Selim H. M. Critical success factors for e-learning acceptance: Confirmatory factor models // Computers \& Education. 2007. Vol. 49. Issue 2.

14. Sufian A. J. Methods and Techniques of Social Research // International journal of business. 2015 . № 2 .

\section{Информация об авторах | Author information}

RU Исаева Елизавета Сергеевна

${ }^{1}$ Южный федеральный университет, г. Ростов-на-Дону

EN Isaeva Elizaveta Sergeevna ${ }^{1}$

${ }^{1}$ Southern Federal University, Rostov-on-Don

${ }^{1}$ isaeva@sfedu.ru

\section{Информация о статье | About this article}

Дата поступления рукописи (received): 29.09.2021; опубликовано (published): 28.12.2021.

Ключевые слова (keywords): дистанционное обучение; система управления обучением; платформа дистанционного образования; онлайн-обучение; distance education; learning management system; distance education platform; online learning. 